\title{
Soft Tissue Sarcomas with Special Reference to Molecular Aberration, Chemotherapy, and Recent Advances: A Review Article
}

\author{
Linu Abraham Jacob ${ }^{1}$ Sreevalli A $^{1}$ (1) Shwetha Ninutha ${ }^{1} \quad$ Lokanatha Dasappa $^{1} \quad$ Suresh Babu MC ${ }^{1}$ \\ Lokesh KN ${ }^{1}$ Rudresha $\mathrm{AH}^{1}$ Rajeev $\mathrm{LK}^{1}$ Smitha Saldanha ${ }^{1}$
}

\author{
${ }^{1}$ Department of Medical Oncology, Kidwai Memorial Institute of \\ Oncology, Bengaluru, India \\ Ind J Med Paediatr Oncol
}

\begin{abstract}
Address for correspondence A Sreevalli, MD, Senior Resident, Department of Medical Oncology, Kidwai Memorial Institute of Oncology, Dr M H, Marigowda Rd, Hombegowda Nagar, Bengaluru, Karnataka- 560029, India (e-mail: svalli88@gmail.com).
\end{abstract}

\begin{abstract}
Keywords

- soft tissue sarcoma

- heterogenous

- mesenchyme

- molecular aberration

Soft tissue sarcomas (STS) are a diverse group of rare solid tumors of mesenchymal cell origin with distinct clinical and pathological features. They account for less than $1 \%$ of all adult malignancies and $15 \%$ of pediatric neoplasms. They include over hundreds of different histological subtypes. Many of these subtypes can occur at any age and are not confined to a specific site. Each subtype displays variable clinical behavior. Low incidence, variable presentation, behavior, and long-term outcomes further make it challenging to treat. There are multiple ongoing trials that focus on the anatomic site and histologic subtype to tailor the treatment. Further rarity of each histotype is a major barrier to recruit patients to randomized controlled trials. A multidisciplinary approach is mandatory in all cases of soft tissue sarcomas.

The purpose of this review is to thoroughly understand the existing literature on history, incidence, epidemiology, etiology, histology, pathogenesis, diagnostic modalities, prognosis, management, and post treatment surveillance of STS. Uterine sarcomas, gastrointestinal stromal tumors (GIST), and pediatric sarcomas are not included here. It briefly highlights various molecular aberrations, changes in staging as per the American Joint Committee on Cancer (AJCC) 8, drugs that are used off-label in specific subtypes of sarcoma along with the recent advances. The classification of STS is undergoing continuous evolution. A wide variety of subtypes can only be diagnosed accurately with sophisticated molecular diagnostic tests and with the involvement of expert geneticists and pathologists to interpret it.

There is no clarity on tailoring the treatment of STS to date. There is always a question on how best we can incorporate chemotherapy and radiotherapy along with surgery as a part of multimodality treatment. The heterogeneity of STS has hindered the development of robust, evidence-based treatment strategies, and our therapeutic approach is neither histology-specific nor widely standardized. Increased knowledge about sarcoma biology could help to discover new and more effective treatment strategies and help overcome the therapeutic challenge imposed by this deadly
\end{abstract}

DOI https://doi.org/ 10.1055/s-0041-1740324. ISSN 0971-5851.

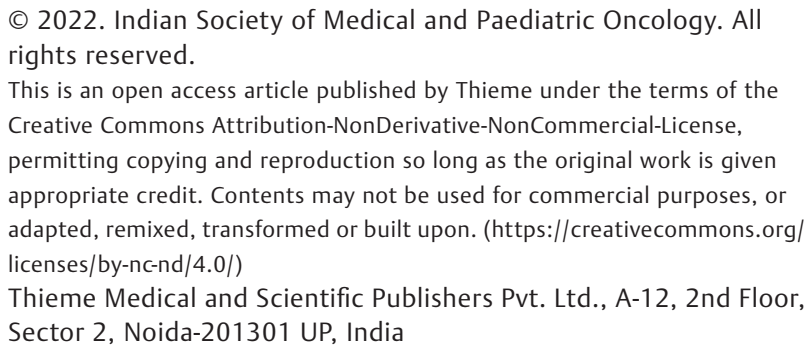

(C) 2022. Indian Society of Medical and Paediatric Oncology. All rights reserved.

This is an open access article published by Thieme under the terms of the Creative Commons Attribution-NonDerivative-NonCommercial-License, permitting copying and reproduction so long as the original work is given appropriate credit. Contents may not be used for commercial purposes, or adapted, remixed, transformed or built upon. (https://creativecommons.org/ licenses/by-nc-nd/4.0/)

Thieme Medical and Scientific Publishers Pvt. Ltd., A-12, 2nd Floor, Sector 2, Noida-201301 UP, India 
disease. Continued collaboration among various sarcoma centers globally will be of prime importance to optimize STS management. This will allow studies to be both sufficiently large and reasonably focused to generate evidence that is clinically meaningful in specific STS patient populations.

\section{Introduction}

Soft tissue sarcomas (STS) are rare and heterogenous group of malignant tumors that originate from embryonic stem cells. Rudolf Virchow, Samuel Gross, and Samuel Wilks laid the foundation of our current understanding of soft tissue sarcomas. ${ }^{1,2}$ They represent a diverse family of malignancies comprising over hundred histological subtypes and many molecular aberrations. ${ }^{3}$ The delay in diagnosis and presentation with advanced disease or metastasis account for poor prognosis. The rarity of the disease along with a wide variety of subtypes has made sarcomas a challenging topic to understand.

\section{Incidence and Epidemiology}

STS comprise less than 1\% of all adult malignancies and 15\% of pediatric malignancies. ${ }^{4}$ As per National Cancer Institute Surveillance Epidemiology End Results (NCI SEER) cancer database, STS accounts for $0.7 \%$ of all new cancer cases with estimated new STS cases in 2020 being 13,130. The life time risk of developing STS is $0.4 \%$. It is most commonly diagnosed in the age group of 65 to 74 years. It represents $0.9 \%$ of all cancer deaths.

The death rate was 1.3 per 100,000 men and women per year. ${ }^{5}$

As per SEER cancer database $60 \%$ of STS are localized at diagnosis, $19 \%$ spread to the regional lymph nodes, $15 \%$ have distant metastasis, and $6 \%$ are of unknown stage. The 5 -year overall relative survival is $64.7 \% .^{5}$ Patients with high-grade tumors are at a significant risk for distant recurrence, and as many as $50 \%$ of these patients die of their disease. ${ }^{6}$ On the contrary, low-grade STS have an excellent prognosis with 5-year survival rates of $85 \%$ or more. ${ }^{7}$

\section{Histologic Subtypes}

The World Health Organization (WHO) 2020 (5 ${ }^{\text {th }}$ edition) classifies STS based on presumptive tissue of origin, architectural pattern if of uncertain histogenesis, and genetics as shown in - Table 1. As per the WHO, there are more than 100 different histologic subtypes. ${ }^{3}$ The most common STS in adults are liposarcoma, followed by leiomyosarcoma, undifferentiated pleomorphic sarcoma, gastrointestinal stromal tumors (GIST), and many others as shown in - Fig. $1 .^{8}$ The Network for Sarcomas (NETSARC) is a large database that enables the exploration of rare clinical and histological subtypes. ${ }^{9}$

\section{Etiology}

The various etiological factors are discussed as below:

\section{Genetic Predisposition}

Genetic syndromes associated with STS are Li-Fraumeni syndrome (LFS), Gardner syndrome (familial adenomatous polyposis), and neurofibromatosis type 1 (NF 1). Mutations in TP53 are the most common germline mutations that predispose to sarcomas such as osteosarcoma, Ewing, and rhabdomyosarcoma. ${ }^{10}$ Sarcomas account for 25 to $33 \%$ of tumors seen in genetic cancer syndromes. ${ }^{11}$

\section{Radiation Therapy and Chemotherapy}

Radiation exposure and risk of STS at 15 to 20 years is almost $0.5 \%{ }^{12}$ The risk is the highest in childhood cancer survivors following RT and chemotherapy with regimens containing anthracyclines and alkylating agents. The most common histopathologic type of radiation-associated sarcoma is undifferentiated pleomorphic sarcoma, previously termed malignant fibrous histiocytoma (MFH). ${ }^{12}$ Undifferentiated pleomorphic sarcoma (UPS) was seen in $26 \%$, angiosarcoma in $21 \%$, fibrosarcoma in $12 \%$, leiomyosarcoma (LMS) in $12 \%$, and malignant peripheral nerve sheath tumor (MPNST) in $9 \%$ of patients. ${ }^{13}$

\section{Chemicals}

Exposure to various chemical agents such as vinyl chloride and arsenic was found to be associated with hepatic angiosarcoma. Other chemicals, exposure to which are at an increased risk of soft tissue sarcoma are thorotrast, dioxin, phenoxy herbicides, and chlorophenol. ${ }^{14}$

\section{Chronic Edema, Chronic Irritation Trauma}

Chronic lymphedema is known to be associated with angiosarcoma. Chronic irritation secondary to foreign bodies and trauma may be responsible for abdominal desmoid. ${ }^{15}$

\section{Viral Infections}

Viral infections such as human immunodeficiency virus (HIV), human herpesvirus-8 (HHV 8), and Epstein-Barr virus (EBV) have also been implicated in the pathogenesis of STS. ${ }^{16}$

\section{Genetics and Molecular Pathogenesis}

The genetics of STS can be divided into those with simple and highly complex karyotypes. Simple karyotype includes fusion genes due to reciprocal translocations or specific point mutations/inversions. Translocation-associated sarcomas 
Table 1 WHO histologic classification of soft tissue sarcomas
A) Fibroblastic/ myofibroblastic tumors
a) Benign
1. Angiomyofibroblastoma
2. Desmoplastic fibroblastoma
3. Myositis ossificans
4. Nodular fasciitis
5. Elastofibroma
6. Fibromatosis colli
7. Angiofibroma NOS
b) Intermediate locally aggressive
1. Solitary fibrous tumor, benign
2. Desmoid - type fibromatoses
3. Palmar/plantar type fibromatoses
c) Intermediate rarely metastasising
1. DFSP - Dermatofibrosarcoma protuberans NOS
2. Inflammatory myofibroblastic tumor
3. Solitary fibrous tumor NOS
4. Infantile fibrosarcoma
5. Superficial CD34-positive fibroblastic tumor
6. Myofibroblastic sarcoma
d) Malignant
1. Solitary fibrous tumor, malignant
2. Fibrosarcoma NOS
3. Myxofibrosarcoma

B) Fibrohistiocytic

a) Benign

1. Tenosynovial giant cell tumor NOS

2. Deep benign fibrous histiocytoma

b) Intermediate (Rarely Metastasizing) Tumors

1. Giant cell tumor of soft part NOS

2. Plexiform fibrohistiocytic tumor

c) Malignant

1. Malignant tenosynovial giant cell tumor

C) Adipocytic

a) Benign

1. Lipoma NOS

2. Lipomatosis

3. Angiomyolipoma NOS

4. Hibernoma intermediate

b) Intermediate

1. Atypical lipomatous tumor / well differentiated liposarcoma

c) Malignant

1. Liposarcoma well differentiated NOS

2. Dedifferentiated liposarcoma

3. Myxoid liposarcoma

4. Pleomorphic liposarcoma

D) Smooth muscle

a) Benign

1. Leiomyoma NOS

b) Malignant

1. Leiomyosarcoma NOS

E) Skeletal muscle

a) Benign

1. Rhabdomyoma NOS

b) Malignant

1. Embryonal rhabdomyosarcoma NOS

2. Alveolar RMS

3. Pleomorphic RMS NOS

4. MYOD1 mutant spindle cell/sclerosing RMS

5. Ectomesenchymoma

F) Vascular

a) Benign

(Continued)

Table 1 (Continued)

1. Hemangioma NOS
2. Lymphangioma NOS
3. Epitheloid hemangioma
b) Intermediate Locally Aggressive
1. Kaposiform hemangioendothelioma
c) Intermediate Rarely Metastasising
1. Kaposi sarcoma
2. Composite hemangioendothelioma
3. Pseudomyogenic hemangioendothelioma
d) Malignant
1. Angiosarcoma
2. Epitheloid hemangioendothelioma NOS with
WWTR1-CAMTA1 fusion
3. Epitheloid hemangioendothelioma with YAP1-TFE3 fusion

G) Perivascular

a) Benign

1. Glomus tumor NOS

2. Angioleiomyoma

3. Myopericytoma

b) Malignant

1. Malignant glomus tumor

H) Neural

a) Benign

1. Neurofibroma NOS

2. Schwannoma NOS

3. Perineuroma NOS

4. Granular cell tumor NOS

5. Benign triton tumor

b) Malignant

1. Malignant peripheral nerve sheath tumor

2. Malignant triton tumor

I) Extraskeletal chondro-osseous tumor

a) Benign

1. Chondroma NOS

2. Chondroblastoma-like soft tissue chondroma

b) Malignant

1. Extraskeletal osteosarcoma

J) Tumors of uncertain differentiation

a) Benign

1. Myxoma NOS

2. Angiomyolipoma

b) Intermediate locally aggressive

1. Hemosiderotic fibrolipomatous tumor

c) Intermediate rarely metastasising

1. Atypical fibroxanthoma

2. Myoepithelioma NOS

d) Malignant

1. Alveolar soft part sarcoma

2. Synovial sarcoma NOS (biphasic, spindle cell, poorly differentiated)

3. Desmoplastic small round cell tumor

4. Epithelioid sarcoma

5. NTRK-rearranged spindle cell neoplasm

6. Clear cell sarcoma NOS

7. Extraskeletal myxoid chondrosarcoma

8. Perivascular epithelioid tumor, malignant

9. Rhabdoid tumor NOS

K) Undifferentiated/ unclassified tumor

a) Malignant

1. Undifferentiated pleomorphic sarcoma

2. Undifferentiated round cell sarcoma

3. Undifferentiated epithelioid sarcoma

4. Undifferentiated sarcoma, not otherwise specified

Abbreviations: NOS, not otherwise Specified; RMS, rhabdomyosarcoma. 
Soft Tissue Sarcoma: A Review Jacob et al.

\section{MOST COMMMON HISTOLOGIC SUBTYPES OF SOFT TISSUE SARCOMAS}

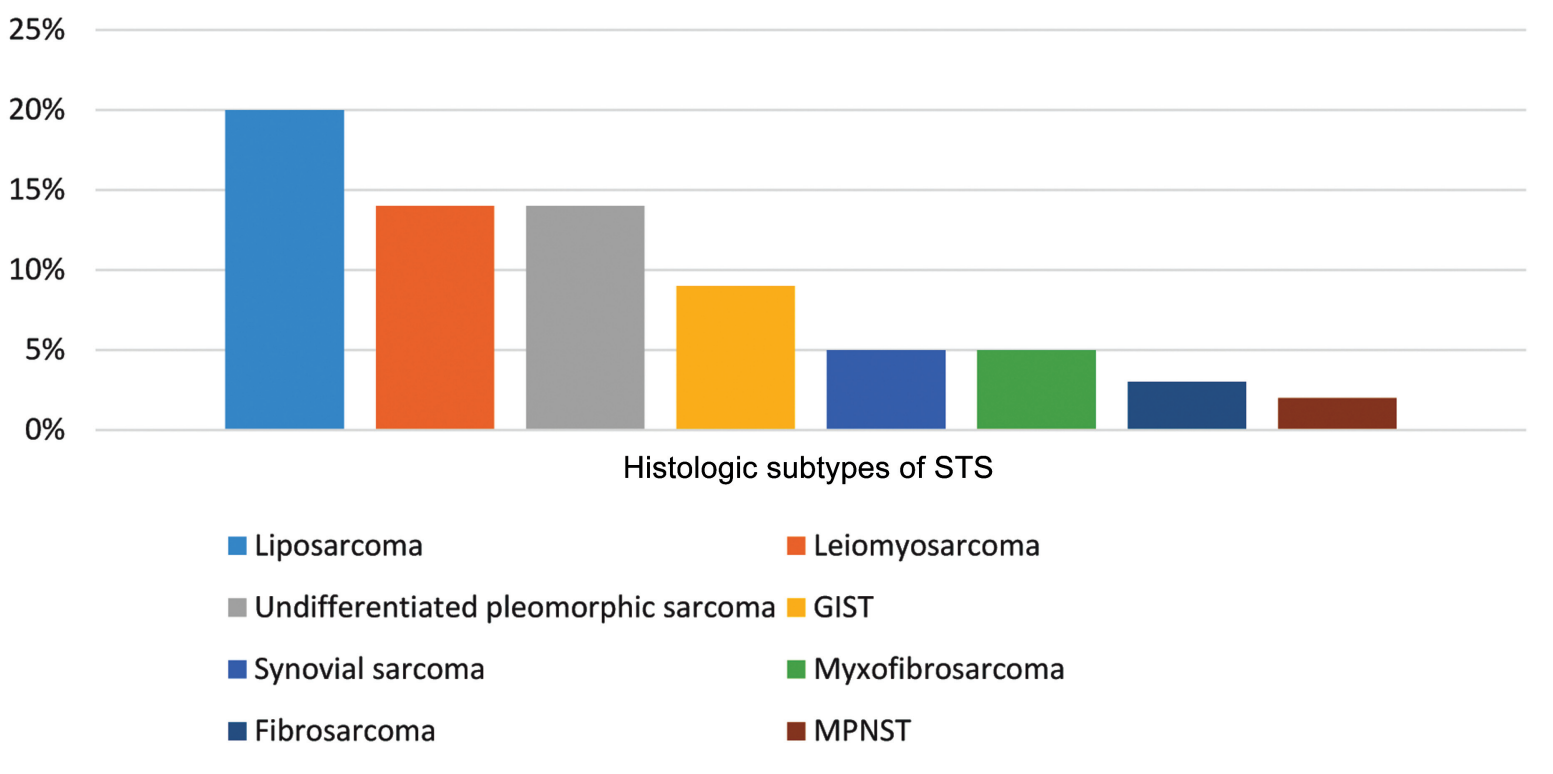

Fig. 1 Histologic subtypes of soft tissue sarcomas with decreasing order of frequency. ${ }^{3}$

Table 2(a) Sarcoma subtypes with associated translocation and genes

\begin{tabular}{|c|c|c|}
\hline Sarcoma Subtype & Translocation & Genes \\
\hline Myxoid/ round cell liposarcoma & $\begin{array}{l}\mathrm{t}(12 ; 16)(\mathrm{q} 13 ; \mathrm{p} 11) \\
\mathrm{t}(12 ; 22)(\mathrm{q} 13 ; \mathrm{q} 12)\end{array}$ & $\begin{array}{l}\text { FUS-DDIT3 }(>90 \%) \\
\text { EWSR1-DDIT3 }(<5 \%)\end{array}$ \\
\hline Ewing sarcoma & $\begin{array}{l}\mathrm{t}(11 ; 22)(\mathrm{q} 24 ; \mathrm{q} 12) \\
\mathrm{t}(21 ; 22)(\mathrm{q} 22 ; \mathrm{q} 12)\end{array}$ & $\begin{array}{l}\text { EWSR1-FLI1 }(>80 \%) \\
\text { EWSR1-ERG }(10-15 \%)\end{array}$ \\
\hline Desmoplastic small round cell tumor & $\mathrm{t}(11 ; 22)(\mathrm{p} 13 ; q 12)$ & EWSR1-WT1 (>75\%) \\
\hline Synovial sarcoma & $\mathrm{t}(\mathrm{x} ; 18)(\mathrm{p} 11 ; \mathrm{q} 11)(>90 \%)$ & $\begin{array}{l}\text { SYT-SSX1 }(66 \%) \\
\text { SYT-SSX2 (33\%) } \\
\text { SYT-SSX4 }(<1 \%)\end{array}$ \\
\hline Alveolar rhabdomyosarcoma & $\begin{array}{l}\mathrm{t}(2 ; 13)(\mathrm{q} 35 ; q 14) \\
\mathrm{t}(1 ; 13)(\mathrm{p} 36 ; \mathrm{q} 14)\end{array}$ & $\begin{array}{l}\text { PAX3-FOXO1 }(<80 \%) \\
\text { PAX7-FOXO1 }(<20 \%) \\
\text { PAX3-NCOA1 }(<1 \%) \\
\text { PAX3-NCOA2 }(<1 \%)\end{array}$ \\
\hline Alveolar soft part sarcoma & $\mathrm{t}(\mathrm{X} ; 17)(\mathrm{p} 11 ; \mathrm{q} 25)$ & ASPSCR1-TFE3(>90\%) \\
\hline $\begin{array}{l}\text { Dermatofibrosarcoma } \\
\text { Protuberans }\end{array}$ & $\begin{array}{l}\mathrm{t}(17 ; 22)(>75 \%) \\
\mathrm{t}(17 ; 22)(\mathrm{q} 22 ; \mathrm{q} 13.1)(10 \%)\end{array}$ & COL1A1-PDGFB \\
\hline Extraskeletal myxoid chondrosarcoma & $\begin{array}{l}\mathrm{t}(9 ; 22)(\mathrm{q} 22 ; \mathrm{q} 12) \\
\mathrm{t}(9 ; 17)(\mathrm{q} 22 ; \mathrm{q} 11) \\
\mathrm{t}(9 ; 15)(\mathrm{q} 22 ; \mathrm{q} 21) \\
\mathrm{t}(3 ; 9)(\mathrm{q} 12 ; \mathrm{q} 22)\end{array}$ & $\begin{array}{l}\text { EWSR1-NR4A3 }(75 \%) \\
\text { TAF15-NR4A3 }(<10 \% \\
\text { TCF12-NR4A3 }(<10 \% \\
\text { TFG-NR4A3 }(<5 \%)\end{array}$ \\
\hline Endometrial stromal tumor & $\mathrm{t}(7 ; 17)(\mathrm{p} 15 ; \mathrm{q} 21)$ & JAZF1-SUZ12 (30\%) \\
\hline Clear cell sarcoma & $\begin{array}{l}\mathrm{t}(12 ; 22)(\mathrm{q} 13 ; \mathrm{q} 12) \\
\mathrm{t}(2 ; 22)(\mathrm{q} 34 ; \mathrm{q} 12)\end{array}$ & $\begin{array}{l}\text { EWSR1-ATF1 }(>75 \%) \\
\text { EWSR1-CREB1 }(<5 \%)\end{array}$ \\
\hline Infantile fibrosarcoma & $\mathrm{t}(12 ; 15)(\mathrm{p} 13 ; \mathrm{q} 25)$ & ETV6-NTRK3 (>75\%) \\
\hline $\begin{array}{l}\text { Inflammatory myofibroblastic } \\
\text { Tumor }\end{array}$ & $\begin{array}{l}\mathrm{t}(1 ; 2)(\mathrm{q} 25 ; \mathrm{p} 23) \\
\mathrm{t}(2 ; 19)(\mathrm{p} 23 ; \mathrm{p} 13) \\
\mathrm{t}(2 ; 17)(\mathrm{p} 23 ; q 23)\end{array}$ & $\begin{array}{l}\text { ALK-TPM34 } \\
\text { ALK-TPM } \\
\text { ALK-CLTC }\end{array}$ \\
\hline Undifferentiated round cell sarcoma & $\begin{array}{l}\mathrm{t}(\mathrm{X} ; \mathrm{X})(\mathrm{p} 11 ; \mathrm{p} 11) \\
\mathrm{t}(4 ; 19)(\mathrm{q} 35 ; \mathrm{q} 13) \\
\mathrm{t}(10 ; 19)(\mathrm{q} 26 ; \mathrm{q} 13)\end{array}$ & $\begin{array}{l}\text { BCOR-CCNB3 } \\
\text { CIC-DUX4 }\end{array}$ \\
\hline
\end{tabular}


Table 2(b) Sarcoma subtypes with associated mutations.

\begin{tabular}{|l|l|l|}
\hline Sarcoma subtype & Mutations & Genes \\
\hline Desmoid fibromatosis & Trisomies 8 and $20(30 \%)$ & $\begin{array}{l}\text { APC inactivation by mutation/deletion } \\
(10 \%) \text { CTNNB1 }(\beta \text {-catenin) mutations }(85 \%)\end{array}$ \\
\hline Embryonal rhabdomyosarcoma & Trisomies 2q, 8, and 20 $(>75 \%)$ & LOH at 11p15 $(>75 \%)$ \\
\hline Solitary fibrous tumor & $12 q 13$ inversion & NAB2-STAT6 $(>95 \%)$ \\
\hline Gastrointestinal stromal tumor & $\begin{array}{l}\text { Monosomies 14 and } \\
22(>75 \%) \\
\text { Deletion of 1p }(>25)\end{array}$ & $\begin{array}{l}\text { KIT or PDGFRA } \\
\text { mutation }(>90 \%)\end{array}$ \\
\hline
\end{tabular}

Abbreviations: APC, adenomatous polyposis coli; LOH, loss of heterozygosity; PDGFRA, platelet-derived growth factor receptor A.

Table 2(c) Sarcoma subtypes with associated complex karyotype

\begin{tabular}{|l|l|l|}
\hline Sarcoma subtype & Complex karyotype & Genes \\
\hline $\begin{array}{l}\text { Well differentiated/ } \\
\text { dedifferentiated liposarcoma }\end{array}$ & $12 q 13-15$ rings(amplification) and giant markers & MDM2 CDK4 \\
\hline Pleomorphic liposarcoma & $\begin{array}{l}13 q 14, \\
17 q 11\end{array}$ & Rb NF1 \\
\hline $\begin{array}{l}\text { Myxofibrosarcoma and undifferentiated } \\
\text { pleomorphic sarcoma }\end{array}$ & $5 p$ amplification & \\
\hline Leiomyosarcoma & del1p, 10q & p53, PTEN, Rb1 \\
\hline Malignant peripheral nerve sheath tumor & $17 q 11$ & NF1, p53 \\
\hline Angiosarcoma & $8 q 24$, & MYC (50\%) \\
\hline & $10 p 12$ & KDR (VEGFR2) \\
\hline
\end{tabular}

Abbreviations: VEGFR2, vascular endothelial growth factor receptor 2.

account for one-third of sarcomas and is more common in young adults 30 to 40 years of age. ${ }^{17}$ Complex karyotype in the form of numerous genetic losses and gains is more common in the 50 to 60 years of age group. The detection of these cytogenetic abnormalities by fluorescence in situ hybridization (FISH) and reverse transcriptase polymerase chain reaction (RT-PCR) can assist in the diagnosis of specific STS as illustrated in - Tables 2(a), 2 (b), $\mathbf{2 ( c ) .}$. $^{18}$

\section{Clinical Features}

The most common clinical presentation is a gradually enlarging painless mass. The most common site of STS is extremities of which the thighs, buttocks, and groin constitute $46 \%$, upper extremity $13 \%$, torso $18 \%$, retroperitoneum $13 \%$, and head and neck $9 \% .{ }^{19}$ Particular histologic subtypes have proclivity to certain anatomic sites, for example liposarcomas (LPS) are more common in the thighs, desmoplastic small round cell tumors mainly involve the abdominal cavity and pelvis. The most common retroperitoneal sarcomas (RPS) are LPS and leiomyosarcomas followed by undifferentiated/unclassified STS. Well-differentiated LPS are the most common followed by dedifferentiated LPS. ${ }^{20}$

\section{Mode of Spread}

The most common mode of spread is hematogenous and mainly to the lungs. According to Christie-Large et al, the incidence of distant metastases at the time of diagnosis of STS was $10 \%$, of which $83 \%$ were located in the lungs. ${ }^{21}$ Other rare sites include the liver, bone, brain, skin, and soft tissues. Extrapulmonary site metastases to retroperitoneum, spine, paraspinous tissues is seen with myxoid/round cell liposarcomas. Overall, $25 \%$ of patients will develop distant metastases after the treatment of primary disease. The chance of distant metastases is almost 40 to $50 \%$ if the tumor size is more than $5 \mathrm{~cm}$, deep to fascia, and intermediate or high grade. $^{22}$

Lymph node spread in STS is very rare and associated with poor prognosis. It is seen more commonly in subtypes such as rhabdomyosarcoma, synovial sarcoma, epithelioid sarcoma, clear cell sarcoma, and angiosarcoma. ${ }^{23}$

\section{Investigations}

Radiological diagnosis is essential to determine the extent of primary, rule out metastases, and plan surgery. Magnetic resonance imaging (MRI) is the investigation of choice for 
Soft Tissue Sarcoma: A Review Jacob et al.

Table 3 FNLCC histologic grading of STS

\begin{tabular}{|l|l|}
\hline & Tumor differentiation \\
\hline 1 & Sarcomas closely resembling normal adult mesenchymal tissue (e.g., low-grade leiomyosarcoma) \\
\hline 2 & Sarcomas of which histologic typing is certain (e.g., myxoid/round cell liposarcoma) \\
\hline & $\begin{array}{l}\text { Embryonal and undifferentiated sarcomas, sarcomas of doubtful type, synovial sarcoma, soft tissue osteosarcoma, } \\
\text { Ewing sarcoma/PNET of soft tissue }\end{array}$ \\
\hline 1 & Mitotic count: In most mitotically active area of the sarcoma, 10 successive HPFs are assessed using a 40x objective \\
\hline 2 & $10-19$ mitosis per 10 HPF \\
\hline 3 & $\geq 20$ mitoses per 10 HPF \\
\hline & Tumor necrosis: evaluated on gross examination \\
\hline 0 & No necrosis \\
\hline 1 & $<50 \%$ tumor necrosis \\
\hline 2 & $\geq 50 \%$ tumor necrosis \\
\hline GX & Grade cannot be assessed \\
\hline G1 & Total differentiation, mitotic count and necrosis score of 2 or 3 \\
\hline G2 & Total differentiation, mitotic count and necrosis score of 4 or 5 \\
\hline G3 & Total differentiation, mitotic count and necrosis score of 6,7 or 8 \\
\hline
\end{tabular}

Abbreviations: HPF, high-power field; PNET, primitive neuroectodermal tumor.

primary extremity lesion and also for detecting local recurrence. ${ }^{24}$ Computerized tomography $(\mathrm{CT})$ is preferred for primary abdominal, visceral, or retroperitoneal lesion. CT chest is required as a part of staging workup to rule out pulmonary metastases. ${ }^{25} \mathrm{CT}$ abdomen and pelvis need to be done in round cell/myxoid liposarcoma due to a high risk of metastasis to the abdomen and retroperitoneum, brain imaging in angiosarcoma and alveolar soft part sarcoma as high chances of brain metastases, bone scan for round cell/myxoid liposarcomas. Positron emission tomography computed tomography (PET CT) is not a part of initial staging workup of STS, including retroperitoneal sarcomas, due to its restricted ability to differentiate benign from low to intermediate grade sarcomas. $^{26}$ As per the National Comprehensive Cancer Network (NCCN), PET scan in STS may be useful for prognostication, grading, and determining the response to neoadjuvant chemotherapy.

Percutaneous core needle biopsy is indicated only if diagnosis is in doubt or if neoadjuvant therapy is planned. ${ }^{27}$ It is rarely performed if initial surgery is planned based on radiographic diagnosis as there is a high chance of tumor seeding. IHC, FISH, RT-PCR, and NGS are the various molecular methods to arrive at final diagnosis. Common IHC markers include NKX2.2 for Ewing sarcoma, CDK4 and MDM2 for well-differentiated/dedifferentiated LPS, TLE-1 for synovial sarcoma, H3K27me3 for MPNST, STAT6 for SFT, EBER-ISH and Pan-NTRK.

\section{Histologic Grading}

The grading of sarcoma includes features such as mitotic index, necrosis, cellularity, pleomorphism, and histologic subtype or differentiation. Among them, the two most important are mitotic index and the extent of necrosis. ${ }^{28}$ Half of retroperitoneal tumors are high grade although this varies according to histology. There are several grading systems of which the two most commonly used are the National Cancer Institute (NCI) system and the FNCLCC system-Federation Nationale des Centres de Lutte Contre le Cancer. The prediction of distant metastasis and tumor mortality was slightly better with the FNCLCC system than the NCI system. ${ }^{29}$ Hence, FNLCC is more commonly used. FNLCC histologic grading is determined by three parameters, i.e., differentiation, mitotic activity, and the extent of necrosis. Each parameter is scored as in - Table 3. The scores are added to determine the grade.

\section{Staging}

The most commonly used staging system for STS is tumor, node, and metastasis (TNM) system, which was developed in collaboration with the Union for International Cancer Control (UICC) with American Joint Committee on cancer (AJCC). The AJCC TNM system uses tumor size (T), lymph node involvement $(\mathrm{N})$, presence or absence of distant metastases $(\mathrm{M})$ along with histologic grading $(\mathrm{G})$ to determine the stage grouping. The recent AJCC 8 determines separate T staging and prognostic stage groups for STS of extremity/trunk and retroperitoneum unlike AJCC 7 as given below in - Table 4(a). It also describes a separate T staging for STS of the abdomen and thorax but no separate $\mathrm{N}$ and $\mathrm{M}$ and prognostic stage groupings (-Table $\mathbf{4}(\mathbf{b})$ ). TNM staging for retroperitoneal sarcomas is same as for the trunk and extremities, except that any T, N1 M0 of any grade is stage IIIB and not stage IV. 
Soft Tissue Sarcoma: A Review Jacob et al.

Table 4(a) AJCC 8 TNM staging/prognostic grouping for soft tissue sarcoma of trunk and extremities

\begin{tabular}{|l|l|}
\hline T & Primary tumor \\
\hline TX & Primary tumor cannot be assessed \\
\hline T0 & No evidence of primary tumor \\
\hline T1 & Tumor $5 \mathrm{~cm}$ or less in greatest dimension \\
\hline T2 & $\begin{array}{l}\text { Tumor more than } 5 \mathrm{~cm} \text { and less than or equal to } \\
10 \mathrm{~cm} \text { in greatest dimension }\end{array}$ \\
\hline T3 & $\begin{array}{l}\text { Tumor more than } 10 \mathrm{~cm} \text { and less than or equal to } \\
15 \mathrm{~cm} \text { in greatest dimension }\end{array}$ \\
\hline T4 & Tumor more than $15 \mathrm{~cm}$ in greatest dimension \\
\hline N & Regional lymph nodes \\
\hline N0 & $\begin{array}{l}\text { No regional lymph node metastasis or unknown } \\
\text { lymph node status }\end{array}$ \\
\hline N1 & Regional lymph node metastasis \\
\hline M & Distant metastases \\
\hline M0 & No distant metastasis \\
\hline M1 & Distant metastasis \\
\hline
\end{tabular}

Table 4(b) AJCC 8 T staging of abdominal and thoracic visceral organs

\begin{tabular}{|l|l|}
\hline T & Primary tumor \\
\hline TX & Primary tumor cannot be assessed \\
\hline T1 & Organ confined \\
\hline T2a & $\begin{array}{l}\text { Tumor invades serosa or visceral } \\
\text { peritoneum }\end{array}$ \\
\hline T2b & $\begin{array}{l}\text { Tumor extends beyond serosa } \\
\text { (mesentery) }\end{array}$ \\
\hline T3 & Invades another organ \\
\hline T4a T4b T4c & $\begin{array}{l}\text { Multifocal involvement (2 sites) multifocal } \\
\text { (3-5 sites) } \\
\text { Multifocal (>5 sites) }\end{array}$ \\
\hline
\end{tabular}

\begin{tabular}{|l|l|l|l|l|}
\hline & T & N & M & G \\
\hline Stage IA & T1 & N0 & M0 & G1, GX \\
\hline Stage IB & T2 & N0 & M0 & G1, GX \\
\hline & T3 & N0 & M0 & G1, GX \\
\hline & T4 & N0 & M0 & G1, GX \\
\hline Stage II & T1 & N0 & M0 & G2, G3 \\
\hline Stage IIIA & N2 & M0 & G2, G3 \\
\hline Stage IIIB & T3 T4 & N0 & M0 & G2, G3 G2, G3 \\
\hline Stage IV & Any T Any T & N1 & M0 M1 & Any G Any G \\
\hline
\end{tabular}

\section{Prognostic Factors}

The most important prognostic factors include histologic grading, tumor size, and pathologic stage at diagnosis. ${ }^{8}$ Others include histologic subtype, anatomic site, depth, patient age, positive margin, recurrent disease at presentation. ${ }^{30}$ Patients with extremity and superficial trunk lesions have better prognosis compared to retroperitoneal and visceral sarcomas.

Grade is an independent predictor of early distant metastases and death. The initial tumor size determines the chances of local recurrence and distant metastases. In a review by Suit et al from Massachusetts General Hospital (MGH), the frequency of distant metastasis increased with an increase in the tumor size, accounting for $38 \%$ of tumors 5$10 \mathrm{~cm}, 49 \%$ of tumors $10.1-15 \mathrm{~cm}, 58 \%$ for tumors $15.1-$ $20 \mathrm{~cm}$, and $83 \%$ for tumors $>20 \mathrm{~cm}$. Bone and neurovascular invasion are associated with bad prognosis.

Grade, completeness of resection, presence or absence of metastatic disease are independent prognostic factors for disease-specific survival in case of retroperitoneal sarcomas. ${ }^{31}$ Patients with MPNST, LMS, high-grade liposarcoma had worse survival compared to solitary fibrous tumor (SFT), low-grade liposarcoma. A nomogram developed by the Memorial Sloan-Kettering Cancer Centre (MSKCC) aids in predicting survival and treatment decision-making for individual patients. It includes patients' age, tumor size, FNLCC grade, histologic subtype, multifocality, and the extent of resection. ${ }^{32}$

\section{Treatment}

\section{- Soft Tissue Sarcoma of Extremities}

The main aim of treating STS of extremities is to improve survival, prevent local and distant recurrences, improve limb function, and minimize morbidity and mortality. The only curative approach is surgical resection. Radiotherapy is indicated for more than $5 \mathrm{~cm}$ tumor to improve local control. The role of chemotherapy in adjuvant and neoadjuvant setting is still controversial. 


\section{Adjuvant Chemotherapy}

Despite 20 randomized controlled trials (RCTs) and two meta-analyses, data on adjuvant chemotherapy are still irreconcilable. As per NCCN and ESMO, adjuvant chemotherapy is an option for high-risk patients-deep-seated STS, high-grade, large primary $\geq 5 \mathrm{~cm}$, locally recurrent extremity sarcoma. Present approach is to individualize treatment based on performance status (PS), age, comorbidity, site of the disease, histology such as synovial sarcoma, leiomyosarcoma, and myxoid round cell liposarcoma, which are chemosensitive.

Sarcoma meta-analysis collaboration (SMAC) in 1997 included 14 RCTs of doxorubicin-based adjuvant chemotherapy (less than 5\% received ifosfamide-containing regimen) after local treatment of 1,568 adults with localized resectable STS. The median follow-up was 9.4 years. It was observed that doxorubicin-based adjuvant chemotherapy significantly improved the time to local recurrence with hazard ratio (HR) of 0.73 (95\% confidence interval [CI]: $0.56-0.94)$ and distant recurrence with $\mathrm{HR}$ of 0.70 (95\%CI: $0.57-0.85$ ), and overall recurrence-free survival (RFS) in adults with HR of 0.75 (95\%CI: 0.64-0.87) and an absolute 6 to $10 \%$ improvement in RFS at 10 years. There was a trend toward OS although not statistically significant. There was no difference in the outcome in relation to various parameters such as age, sex, stage, site, grade, histology, extent of resection, tumor size, or exposure to radiotherapy. However, on subset analysis those with extremity and truncal sarcomas had significant OS benefit. There was almost 7\% absolute benefit in OS at 10 years in those receiving doxorubicinbased regimens, which was statistically significant. ${ }^{33,34}$

However, the main drawback of this meta-analysis was it included only one negative trial of the two European organizations for research and treatment of cancer (EORTC) negative trials.

Petrioli et al in an Italian trial included 88 patients with high-risk extremity sarcoma were randomized to surgery with or without RT and surgery with or without RT with chemotherapy (epirubicin or IE regimen). The 5-year disease-free survival (DFS) and OS were higher in chemotherapy arm, which was statistically significant accounting for $69 \%$ versus $44 \%$ and $72 \%$ versus $47 \%$, respectively. ${ }^{35}$ This study highlights the advantage of epirubicin-based adjuvant chemotherapy in high-risk STS.

EORTC and an Austrian trial could not demonstrate the survival benefit from adjuvant doxorubicin- and ifosfamidecontaining regimens. ${ }^{36,37}$ EORTC included 351 patients with completely resected STS, of which $67 \%$ were extremity tumors, $60 \%$ were high grade, and $40 \%$ were $\geq 10 \mathrm{~cm}$ in size. There was no difference in RFS and OS in chemotherapy (five cycles of ifosfamide $5 \mathrm{gm} / \mathrm{m}^{2}$ cycle and adriamycin $75 \mathrm{mg} / \mathrm{m}^{2}$-IA) and observation arm. ${ }^{36}$ The limitations of these studies were small number of patients, admittance of non-extremity, small and low/intermediate-grade primaries, and the use of relatively low ifosfamide dose.

Pervaiz et al in 2008 demonstrated the benefit of chemotherapy in an updated systematic meta-analysis (SMAC) of
18 RCTs. It included negative trials such as Austrian and two Italian trials but not the EORTC trial. A total of 1,953 patients with localized surgically amenable STS were included between 1973 and 2002 (five trials used IA regimen, while others used either doxorubicin alone or a combination regimen). The odds ratio (OR) for local and distant recurrence favored chemotherapy similar to the SMAC meta-analysis. When compared to the SMAC meta-analysis, doxorubicin and ifosfamide were associated with statistically significant OS benefit (HR for death: $0.56,95 \% \mathrm{CI}$ : $0.36-0.85$ ) with an absolute risk reduction accounting to $11 \%$ (30 vs. $41 \%$ risk of death). However, the benefit could not be shown for doxorubicin alone. Thus, ifosfamide is of utmost importance in adjuvant setting of sarcomas.

— Role of tumor histology, grade, and size in adjuvant therapy

There are no prospective trials to prove the same. Three retrospective studies have showed benefit of adjuvant chemotherapy in chemosensitive subtypes such as myxoid round cell liposarcoma and synovial sarcoma. In a single center Italian trial of 251 patients with localized synovial sarcoma, 61 patients who had undergone macroscopically complete resection, received adjuvant chemotherapy. The 5year metastasis-free survival (MFS) in chemotherapy arm was $60 \%$ as against $48 \%$ in surgery only arm. The benefit was more for those older than 17 years and $\geq 5 \mathrm{~cm}$ tumor size (MFS: 47 vs. $27 \%$, respectively). ${ }^{38}$ However, as per both MSKCC and MD Anderson retrospective data between 1984 and 1999, patients undergoing adjuvant chemotherapy for high-grade, $\geq 5 \mathrm{~cm}$ extremity STS did not show any benefit. ${ }^{39}$

The optimal regimen is unspecified- the AIM regimen consisting of adriamycin $75 \mathrm{mg} / \mathrm{m}^{2}$, ifosfamide $9-10 \mathrm{gm} / \mathrm{m}^{2}$ D1-D3 with mesna every three weekly for a total of six cycles is used commonly.

\section{Neoadjuvant Chemotherapy}

The role of neoadjuvant therapy is in large, recurrent, and high-grade tumors where limb salvage is an issue. The correct sequencing of chemotherapy (CT), RT, and surgery is unknown. The main purpose of neoadjuvant therapy is the treatment of micro metastases, need for less radical surgery, and more effectiveness of preop RT or chemotherapy. There are no randomized phase III trials to support neoadjuvant chemotherapy. A randomized phase II EORTC study of 150 patients randomizing them to three cycles of neoadjuvant ifosfamide and adriamycin versus surgery alone was negative.

\section{n Role of histology in neoadjuvant therapy}

In a phase III multicentric randomized trial, as compared to histology-based treatment with high-dose ifosfamide alone for synovial sarcoma, gemcitabine plus dacarbazine for LMS, gemcitabine plus docetaxel for undifferentiated pleomorphic sarcoma, trabectedin for high-grade myxoid liposarcoma, etoposide plus ifosfamide for MPNST, anthracycline-based regimen (IE regimen) showed DFS benefit among all histologies including LMS and MPNST. Data are still not convincing as to how epirubicin influenced the trial 
results as doxorubicin is more commonly used in all other trials.

\section{- Retroperitoneal Sarcomas}

The only curative treatment for RPS has been surgery. The ability to achieve R0/R1 resection at the time of initial presentation is the most important prognostic factor for survival. Debulking surgery (R2 resection) should be preferred only in large unresectable well-differentiated retroperitoneal liposarcomas, which aids in symptom improvement and prolongs survival.

Intra-op radiotherapy (IORT) of 10 to 15 Gy can be administered to the areas of residual microscopic or gross disease as it improves local control, RFS, and OS. However, no RCTs are available to support the same. ${ }^{40}$ Reresection is advised for gross residual disease ( $\mathrm{R} 2$ resection), if technically feasible.

\section{Adjuvant Therapy}

Evidence for adjuvant therapy for RPS, may it be RT and/or chemotherapy, is lacking. Adjuvant RT is indicated for intermediate- to high-grade tumors and incompletely resected tumors at high risk for recurrence. Survival benefit has been demonstrated in retrospective and case-control studies. ${ }^{41}$ Postoperative RT enhances local tumor control and has shown a trend toward long-term RFS. However, the management of radiation-associated morbidity is challenging. Hence, safe delivery of RT is feasible preoperatively than postop. Anthracycline/ifosfamide-containing regimen significantly improved the survival as per an updated metaanalysis but it did not include two negative trials. ${ }^{42,43}$

\section{Neoadjuvant Therapy}

Data on neoadjuvant chemotherapy for RPS are very limited. As per the ESMO guidelines tailoring treatment is based on histology and grade. In case of large well-differentiated liposarcoma (WD LPS), intermediate-and high-grade tumors that are not chemosensitive or patient not suitable for chemotherapy neoadjuvant RT, followed by a surgery is preferred. Pre-op RT permits safe delivery of higher doses than in post-op setting and is biologically more effective; however, no RCTs are available as yet to support the same in RPS. Results of STRASS I, a randomized EORTC phase III trial is awaited. ${ }^{44}$ Intensity modulated radiotherapy (IMRT) has the ability to improve the therapeutic index; however, longer follow-up is needed. ${ }^{45}$
There are no randomized control trials on concurrent chemoradiation. Smaller studies have been done in cases of leiomyosarcoma, undifferentiated pleomorphic sarcoma but longterm outcomes are not reported.

Neoadjuvant CT with or without perioperative RT would be an option for intermediate- and high-grade tumors with chemosensitive histology such as synovial sarcoma, myxoid/round cell, LPS and those with high risk of distant metastases such as LMS, large undifferentiated LPS (STRASS2 trial). ${ }^{46}$ Data on optimal regimen to be chosen for RPS are lacking. Histotype-driven treatment when compared to ifosfamide and adriamycin regimen in high-risk STS did not show any significant benefit. Patients with high-risk tumors may benefit from regional hyperthermia along with systemic chemotherapy (EORTC study 62961). ${ }^{47}$ However, data on whether this approach is superior to surgery, with or without RT are lacking.

\section{- Metastatic STS}

Once distant metastases develop, the median survival is 12 to 19 months. Surgical resection of isolated pulmonary metastatic disease results in long-term RFS with 5-year survival of 25 to $40 \% .{ }^{48}$ In patients with unresectable disease, around 20 to $25 \%$ are still alive at 2 to 3 years. ${ }^{49}$ However, the survival is dependent on disease biology, histology, and treatment received.

For asymptomatic, low-grade, unresectable tumors such as low-grade intra-abdominal LMS, intra-abdominal WDLPS, follow-up may be reasonable. Patients with good performance status (PS), minimal comorbidity with anthracycline sensitive high-grade histologies (-Table 5), chemotherapy with doxorubicin with or without ifosfamide is preferable.

Combination chemotherapy with doxorubicin ifosfamide instead of single agent doxorubicin is chosen for symptomatic patients who require rapid tumor response. Gemcitabine-based chemotherapy is preferred for those with contraindication to anthracyclines such as cardiac failure, who have received doxorubicin cumulative dose of $\geq 375$ $\mathrm{mg} / \mathrm{m}^{2} .{ }^{50}$ Paclitaxel is an advisable alternative to anthracyclines for initial treatment of angiosarcoma. Various combination regimens available are doxorubicin plus ifosfamide with mesna (AIM), or AIM with dacarbazine (MAID), gemcitabine plus either docetaxel, vinorelbine, or dacarbazine, doxorubicin plus dacarbazine. Combination regimens are associated with higher response rates (18-46\%) when compared to single-agent doxorubicin (12-18\%)..$^{51-53}$

Table 5 Tumor sensitivity to anthracycline

\begin{tabular}{|l|l|}
\hline Anthracycline-sensitive histology & Anthracycline-resistant histology \\
\hline Leiomyosarcoma Myxoid/round cell liposarcoma & Alveolar soft part sarcoma Extraskeletal myxoid chondrosacroma \\
Dedifferentiated and pleomorphic liposarcoma & Solitary fibrous tumor Hemangiopericytoma \\
Synovial sarcoma Epithelioid sarcoma (ES) & PEComas: angiomyolipoma/lymphangioleiomyomatosis Tenosynovial \\
Angiosarcoma & giant cell tumor (TGCT) \\
Undifferentiated pleomorphic sarcoma & Clear cell sarcoma \\
Malignant pleomorphic sarcoma & Dermatofibrosarcoma protruberans (DFSP) \\
\hline
\end{tabular}

Abbreviations: DFSP, dermatofibrosarcoma protruberans; ES, epithelioid sarcoma; PEComas, perivascular epithelioid cell tumor; TGCT, tenosynovial giant cell tumor. 
Patients with poor PS, multiple comorbidities singleagent gemcitabine, pegylated liposomal doxorubicin are the options. Single-agent doxorubicin, epirubicin, and ifosfamide provide response rates (RR) of more than $20 \%$ in metastatic STS. ${ }^{17}$ Other single agents such as vinorelbine, dacarbazine, temozolomide particularly for leiomyosarcomas have a RR less than $20 \%{ }^{54}$

For patients with metastatic or advanced STS with anthracycline-resistant histologies, the following are the options:

> Pazopanib or sunitinib can be used for alveolar soft part sarcoma or extraskeletal myxoid chondrosarcoma with symptomatic or progressive disease. Phase II elderly patients with metastatic or advanced soft tissue sarcoma (EPAZ), a trial showed that pazopanib was noninferior to doxorubicin in terms of PFS (median PFS 4.4 vs. 5.3 months, HR: $1.00,95 \% \mathrm{CI}$ : $0.65-1.53$ ) with similar OS. ${ }^{55}$

$>$ Dacarbazine with or without doxorubicin, temozolomide plus bevacizumab, pazopanib or sunitinib are the options for SFT/hemangiopericytoma. ${ }^{55}$

$>$ Imatinib is an alternative for locally advanced, metastatic, or recurrent dermatofibrosarcoma protruberans (DFSP) as per a systematic review (complete response $[\mathrm{CR}]$ in $5.2 \%$ and partial response [PR] in $55.2 \%) .{ }^{56}$

> Sirolimus can be chosen for symptomatic progressive, recurrent perivascular epithelioid cell differentiation PEComas including angiomyolipoma/ lymphangioleiomyomatosis. ${ }^{57}$

$>$ Pexidartanib colony-stimulating factor 1 receptor (CSF1R) inhibitor is an option for unresectable, recurrent, or relapsed tenosynovial giant cell tumor (TGCT) based on positive results from phase III ENLIVEN trial. $^{58}$

> Cediranib has substantial single-agent activity in metastatic alveolar soft part sarcoma (ASPS) with an ORR of $35 \%$ and a disease control rate of $84 \%$ at 24 weeks. ${ }^{59}$

\section{- Options for Second Line}

For patients with good PS who progress on doxorubicinbased first line, later therapies are based on histology, which may include

$>$ Gemcitabine docetaxel combination regimen for LMS, MFH. ${ }^{60}$ PLD, gemcitabine alone or combination regimens, ifosfamide alone or with doxorubicin are the other chemotherapy options. ${ }^{61}$

> Trabectadine is an option for advanced LMS, myxoid/ round cell liposarcoma and translocation-related sarcomas based on phase III, T-SAR trial with OS and PFS benefit in those previously treated with anthracycline-based chemotherapy when compared to dacarbazine. ${ }^{62}$ Threeweekly regimen was superior to weekly dosing.

$>$ Eribulin can be preferred over trabectadine for pleomorphic and dedifferentiated liposarcomas due to better OS and PFS and similar PFS and OS in LMS when compared to dacarbazine. ${ }^{63}$

$>$ Pazopanib is an alternative for non-adipocytic advanced sarcomas-mainly LMS, synovial sarcoma, angiosarcoma, SFT. Phase III PALETTE trial showed a significant PFS benefit (median PFS 4.6 vs. $1.6 \mathrm{~m}$ ) when compared to placebo. ${ }^{64}$

> Among patients with progressive, refractory, or symptomatic desmoid tumors, sorafenib significantly prolonged PFS and induced durable responses in a phase III trial. $^{65}$

$>$ Larotrectinib or entrectinib could be an option for tumors with gene fusions involving one of neurotrophic tyrosine receptor kinase (NTRK) genes. ALKA, STARTRK-1, and STARTRK-2 trials demonstrated an ORR of $46 \%$ with entrectinib and $75 \%$ with larotrectinib. $^{66,67}$

> Pembrolizumab is an alternative for microsatellite instability (MSI) high/mismatch repair-deficient STS. ${ }^{68}$

\section{Recent Advances}

$\checkmark$ Doxorubicin with olaratumab in phase III ANNOUNCE trial showed an inferior PFS and hence olaratumab has been withdrawn from the market ${ }^{68}$.

$\checkmark$ In a phase III trial evofosfamide in combination with doxorubicin compared to doxorubicin alone in previously untreated patients with locally advanced, unresectable, or metastatic STS showed no significant difference in median OS and PFS. ${ }^{69}$

Aldoxorubicin, a tumor-targeted doxorubicin conjugate, in a phase III study, showed significant PFS (5.3 vs. 2.9 months) and disease control rate (41.7 vs. $27 \%$ ) in a subcohort of L-sarcomas. ${ }^{70}$

$\checkmark$ Tazemostat, first and only Enhancer of zeste homolog (EZH2) inhibitor-an epigenetic regulator-was approved by the Food and Drug Administration (FDA) for advanced, recurrent metastatic epithelioid sarcoma (ES) not amenable for resection on Jan 23, 2020, based on a phase II trial. ${ }^{69}$

$\checkmark$ Pazopanib can be used as an initial therapy in anthracycline-sensitive advanced STS histologies for elderly who may not tolerate doxorubicin (October 2020, phase II study). ${ }^{55}$

$\checkmark$ Crizotinib in phase I/II trial, demonstrated CR and PR in $36 \%$ and $50 \%$ of inflammatory myofibroblastic tumor (IMT) with ALK translocation. More recently, ceritinib was thought to improve disease-free interval in those with acquired resistance to crizotinib. $^{71}$

$\checkmark$ Anlotinib in a phase II study showed a statistically significant PFS benefit when compared to placebo in metastatic STS who had failed standard chemotherapy (China FDA in 2019). ${ }^{72}$

$\checkmark$ Maintenance ridaforolimus for advanced STS in a phase III study showed $28 \%$ reduction in PFS after four cycles of chemotherapy (SUCCEED). ${ }^{70}$

$\checkmark$ Palbociclib and abemaciclib in several phase I/II trials was associated with a favorable PFS of $66 \%$ in patients with cyclin-dependent kinase 4 (CDK4)-amplified WDLS/DDLS who had progressive disease despite systemic therapy. ${ }^{73}$ 
Selinexor, an oral selective inhibitor of nuclear exportin protein in phase II/III trial (SEAL) for patients with DDLS showed improved PFS. Currently, recruitment of patients for phase III portion is ongoing. ${ }^{74}$

\section{Follow-Up}

As per NCCN and ESMO, follow-up is recommended every three to six monthly with history and physical examination for 2 to 3 years, then every 6 months for next 2 years, and then annually for 10 years. Periodic imaging of the primary site and CT chest for histologies with high chance of pulmonary metastases is recommended on follow-up. ${ }^{75}$

\section{Conclusion}

Heterogeneity of STS makes it a challenging rare malignancy to treat. The disease has got diverse outcomes determined by numerous factors. Prognostic factors and molecular aberrations should be taken into account while tailoring the treatment plan. A multidisciplinary approach is warranted. Many randomized prospective trials including patients with a single histological subtype of STS are needed due to heterogeneity across the histological subtypes and also in terms of chemosensitivity to formulate uniform guidelines for management of the same.

\section{Authors' Contributions}

LAJ, SA, SN, and LD contributed to the conception of the study. SA, LAJ, LD, and SS were responsible for the acquisition. SA, LAJ, SBMC, LKN, AHR, and LKR drafted the manuscript. LD, LAJ, and LKR substantively revised it. All authors have read and approved the manuscript.

\section{Funding}

None.

\section{Conflict of Interest}

None declared.

\section{Acknowledgments}

We would like to thank the residents and faculty of the Department of Medical Oncology, Kidwai Memorial Institute of Oncology, Bangalore.

\section{References}

1 Gross SD. (Samuel D). A System of Surgery: Pathological, Diagnostic, Therapeutic, and Operative. Philadelphia: H.C. Lea; 1866

2 VIRCHOW. Rudolf (1821-1902). Die Cellularpathologie in ihrer Begründung auf physiologische und pathologische Gewebelehre. Berlin: August Hirschwald; 1858

3 Fletcher CDM BJ, Hogendoorn PCW, Mertens F. WHO Classification of Tumours of Soft Tissue and Bone. 4th ed. Lyon: IARC Press; 2013

4 Siegel RL, Miller KD, Jemal A. Cancer statistics, 2019. CA Cancer J Clin 2019;69(01):7-34

5 Cancer of soft tissue including heart - Cancer Stat Facts. SEER. Accessed September 18, 2020 at: https://seer.cancer.gov/statfacts/html/soft.html
6 Kattan MW, Leung DHY, Brennan MF. Postoperative nomogram for 12-year sarcoma-specific death. J Clin Oncol 2002;20(03):791-796

7 Marcus SG, Merino MJ, Glatstein E, et al. Long-term outcome in 87 patients with low-grade soft-tissue sarcoma. Arch Surg 1993;128 (12):1336-1343

8 Zagars GK, Ballo MT, Pisters PWT, et al. Prognostic factors for patients with localized soft-tissue sarcoma treated with conservation surgery and radiation therapy: an analysis of 1225 patients. Cancer 2003;97(10):2530-2543

9 Blay J-Y, Toulmonde M, Penel N, et al. Natural history of sarcomas and impact of reference centers in the nationwide NETSARC study on 35,784 patients (pts) from 2010 to 2017. Ann Oncol 2018;29 (08): viii576-viii595. 10.1093/annonc/mdy299

10 Carnevale A, Lieberman E, Cárdenas R. Li-Fraumeni syndrome in pediatric patients with soft tissue sarcoma or osteosarcoma. Arch Med Res 1997;28(03):383-386

11 Mai PL, Best AF, Peters JA, et al. Risks of first and subsequent cancers among TP53 mutation carriers in the National Cancer Institute Li-Fraumeni syndrome cohort. Cancer 2016;122(23): 3673-3681

12 Brady MS, Gaynor JJ, Brennan MF. Radiation-associated sarcoma of bone and soft tissue. Arch Surg 1992;127(12):1379-1385

13 Torres KE, Ravi V, Kin K, et al. Long-term outcomes in patients with radiation-associated angiosarcomas of the breast following surgery and radiotherapy for breast cancer. Ann Surg Oncol 2013; 20(04):1267-1274

14 Kogevinas M, Becher H, Benn T, et al. Cancer mortality in workers exposed to phenoxy herbicides, chlorophenols, and dioxins. An expanded and updated international cohort study. Am J Epidemiol 1997;145(12):1061-1075

15 Stewart FW, Treves N. Classics in oncology: lymphangiosarcoma in postmastectomy lymphedema: a report of six cases in elephantiasis chirurgica. CA Cancer J Clin 1981;31(05):284-299

16 Purgina B, Rao UNM, Miettinen M, Pantanowitz L. AIDS-related EBV-associated smooth muscle tumors: a review of 64 published cases. Patholog Res Int. 2011. Doi: 10.4061/2011/561548

17 Borden EC, Baker LH, Bell RS, et al. Soft tissue sarcomas of adults: state of the translational science. Clin Cancer Res 2003;9(06): 1941-1956

18 Italiano A, Di Mauro I, Rapp J, et al. Clinical effect of molecular methods in sarcoma diagnosis (GENSARC): a prospective, multicentre, observational study. Lancet Oncol 2016;17(04):532-538

19 Lawrence W Jr, Donegan WL, Natarajan N, Mettlin C, Beart R, Winchester D. Adult soft tissue sarcomas. A pattern of care survey of the American College of Surgeons. Ann Surg 1987;205(04): 349-359

20 Pham TH, Iqbal CW, Zarroug AE, Donohue JH, Moir C. Retroperitoneal sarcomas in children: outcomes from an institution. J Pediatr Surg 2007;42(05):829-833

21 Christie-Large M, James SLJ, Tiessen L, Davies AM, Grimer RJ. Imaging strategy for detecting lung metastases at presentation in patients with soft tissue sarcomas. Eur J Cancer 2008;44(13): 1841-1845

22 Weitz J, Antonescu CR, Brennan MF. Localized extremity soft tissue sarcoma: improved knowledge with unchanged survival over time. J Clin Oncol 2003;21(14):2719-2725

23 Fong Y, Coit DG, Woodruff JM, Brennan MF. Lymph node metastasis from soft tissue sarcoma in adults. Analysis of data from a prospective database of 1772 sarcoma patients. Ann Surg 1993; 217(01):72-77

24 Aisen AM, Martel W, Braunstein EM, McMillin KI, Phillips WA, Kling TF. MRI and CT evaluation of primary bone and soft-tissue tumors. Am J Roentgenol 1986;146(04):749-756

25 Casali PG, Abecassis N, Bauer S, et al. Soft tissue and visceral sarcomas: ESMO- EURACAN Clinical Practice Guidelines for diagnosis, treatment and follow-up†. Ann Oncol 2018;29: iv51-iv67.6 
26 Folpe AL, Lyles RH, Sprouse JT, Conrad EU III, Eary JF. (F-18) fluorodeoxyglucose positron emission tomography as a predictor of pathologic grade and other prognostic variables in bone and soft tissue sarcoma. Clin Cancer Res 2000;6(04):1279-1287

27 Strauss DC, Qureshi YA, Hayes AJ, Thway K, Fisher C, Thomas JM. The role of core needle biopsy in the diagnosis of suspected soft tissue tumours. J Surg Oncol 2010;102(05):523-529

28 World Health Organization. Classification of tumours. In: Fletcher CDM, Unni KK, Mertens F, eds. Pathology and Genetics of Tumors of Soft Tissue. Lyon: IARC Press; 2002:12-224

29 Guillou L, Coindre JM, Bonichon F, et al. Comparative study of the National Cancer Institute and French Federation of Cancer Centers Sarcoma Group grading systems in a population of 410 adult patients with soft tissue sarcoma. J Clin Oncol 1997;15(01): 350-362

30 Pisters PW, Leung DH, Woodruff J, Shi W, Brennan MF. Analysis of prognostic factors in 1,041 patients with localized soft tissue sarcomas of the extremities. J Clin Oncol 1996;14(05):1679-1689

31 Tan MCB, Brennan MF, Kuk D, et al. Histology-based classification predicts pattern of recurrence and improves risk stratification in primary retroperitoneal sarcoma. Ann Surg 2016;263(03): 593-600

32 Gronchi A, Miceli R, Shurell E, et al. Outcome prediction in primary resected retroperitoneal soft tissue sarcoma: histology-specific overall survival and disease-free survival nomograms built on major sarcoma center data sets. J Clin Oncol 2013;31(13): 1649-1655

33 Sarcoma Meta-analysis Collaboration. Adjuvant chemotherapy for localised resectable soft-tissue sarcoma of adults: meta-analysis of individual data. Lancet 1997;350(9092):1647-1654

34 Sarcoma Meta-analysis Collaboration (SMAC) Adjuvant chemotherapy for localised resectable soft tissue sarcoma in adults. Cochrane Database Syst Rev 2000;(02):CD001419

35 Petrioli R, Coratti A, Correale P, et al. Adjuvant epirubicin with or without Ifosfamide for adult soft-tissue sarcoma. Am J Clin Oncol 2002;25(05):468-473

36 Woll PJ, Reichardt P, Le Cesne A, et al; EORTC Soft Tissue and Bone Sarcoma Group and the NCIC Clinical Trials Group Sarcoma Disease Site Committee. Adjuvant chemotherapy with doxorubicin, ifosfamide, and lenograstim for resected soft-tissue sarcoma (EORTC 62931): a multicentre randomised controlled trial. Lancet Oncol 2012;13(10):1045-1054

37 Brodowicz T, Schwameis E, Widder J, et al. Intensified adjuvant IFADIC chemotherapy for adult soft tissue sarcoma: a prospective randomized feasibility trial. Sarcoma 2000;4(04):151-160

38 Ferrari A, Gronchi A, Casanova M, et al. Synovial sarcoma: a retrospective analysis of 271 patients of all ages treated at a single institution. Cancer 2004;101(03):627-634

39 Cormier JN, Huang X, Xing Y, et al. Cohort analysis of patients with localized, high-risk, extremity soft tissue sarcoma treated at two cancer centers: chemotherapy-associated outcomes. J Clin Oncol 2004;22(22):4567-4574

40 Petersen IA, Haddock MG, Donohue JH, et al. Use of intraoperative electron beam radiotherapy in the management of retroperitoneal soft tissue sarcomas. Int J Radiat Oncol Biol Phys 2002;52 (02):469-475

41 Nussbaum DP, Rushing CN, Lane WO, et al. Preoperative or postoperative radiotherapy versus surgery alone for retroperitoneal sarcoma: a case-control, propensity score-matched analysis of a nationwide clinical oncology database. Lancet Oncol 2016;17 (07):966-975

42 Pervaiz N, Colterjohn N, Farrokhyar F, Tozer R, Figueredo A, Ghert M. A systematic meta-analysis of randomized controlled trials of adjuvant chemotherapy for localized resectable soft-tissue sarcoma. Cancer 2008;113(03):573-581

43 Le Cesne A, Ouali M, Leahy MG, et al. Doxorubicin-based adjuvant chemotherapy in soft tissue sarcoma: pooled analysis of two STBSGEORTC phase III clinical trials. Ann Oncol 2014;25(12):2425-2432
44 Bonvalot S, Gronchi A, Le Pechoux C, et al. STRASS (EORTC 62092): a phase III randomized study of preoperative radiotherapy plus surgery versus surgery alone for patients with retroperitoneal sarcoma. J Clin Oncol 2019;37(15, suppl):11001-11001

45 Bossi A, De Wever I, Van Limbergen E, Vanstraelen B. Intensity modulated radiation-therapy for preoperative posterior abdominal wall irradiation of retroperitoneal liposarcomas. Int J Radiat Oncol Biol Phys 2007;67(01):164-170. Erratum in: Int J Radiat Oncol Biol Phys. 2007 May 1;68(1):317. Dosage error in article text.

46 van Houdt WJ, Raut CP, Bonvalot S, Swallow CJ, Haas R, Gronchi A. New research strategies in retroperitoneal sarcoma. The case of TARPSWG, STRASS and RESAR: making progress through collaboration. Curr Opin Oncol 2019;31(04):310-316

47 Issels RD, Lindner LH, Verweij J, et al; European Organisation for Research and Treatment of Cancer Soft Tissue and Bone Sarcoma Group (EORTC-STBSG) European Society for Hyperthermic Oncology (ESHO) Neo-adjuvant chemotherapy alone or with regional hyperthermia for localised high-risk soft-tissue sarcoma: a randomised phase 3 multicentre study. Lancet Oncol 2010;11(06): 561-570

48 Choong PF, Pritchard DJ, Rock MG, Sim FH, Frassica FJ. Survival after pulmonary metastasectomy in soft tissue sarcoma. Prognostic factors in 214 patients. Acta Orthop Scand 1995;66(06): 561-568

49 Billingsley KG, Burt ME, Jara E, et al. Pulmonary metastases from soft tissue sarcoma: analysis of patterns of diseases and postmetastasis survival. Ann Surg 1999;229(05):602-610, discussion 610-612

50 Merimsky O, Meller I, Flusser G, et al. Gemcitabine in soft tissue or bone sarcoma resistant to standard chemotherapy: a phase Il study. Cancer Chemother Pharmacol 2000;45(02):177-181

51 Santoro A, Tursz T, Mouridsen H, et al. Doxorubicin versus CYVADIC versus doxorubicin plus ifosfamide in first-line treatment of advanced soft tissue sarcomas: a randomized study of the European Organization for Research and Treatment of Cancer Soft Tissue and Bone Sarcoma Group. J Clin Oncol 1995;13(07):1537-1545

52 Antman K, Crowley J, Balcerzak SP, et al. An intergroup phase IIl randomized study of doxorubicin and dacarbazine with or without ifosfamide and mesna in advanced soft tissue and bone sarcomas. J Clin Oncol 1993;11(07):1276-1285

53 Hensley ML, Maki R, Venkatraman E, et al. Gemcitabine and docetaxel in patients with unresectable leiomyosarcoma: results of a phase II trial. J Clin Oncol 2002;20(12):2824-2831

54 Borden EC, Amato DA, Rosenbaum C, et al. Randomized comparison of three adriamycin regimens for metastatic soft tissue sarcomas. J Clin Oncol 1987;5(06):840-850

55 Grünwald V, Karch A, Schuler M, et al. Randomized comparison of pazopanib and doxorubicin as first-line treatment in patients with metastatic soft tissue sarcoma age 60 years or older: results of a German Intergroup Study. J Clin Oncol 2020;38(30):3555-3564

56 Navarrete-Dechent C, Mori S, Barker CA, Dickson MA, Nehal KS. Imatinib treatment for locally advanced or metastatic dermatofibrosarcoma protuberans: a systematic review. JAMA Dermatol 2019;155(03):361-369

57 Folpe AL, Mentzel T, Lehr H-A, Fisher C, Balzer BL, Weiss SW. Perivascular epithelioid cell neoplasms of soft tissue and gynecologic origin: a clinicopathologic study of 26 cases and review of the literature. Am J Surg Pathol 2005;29(12):1558-1575

58 Lamb YN. Pexidartinib: First Approval. Drugs 2019;79(16): 1805-1812

59 Kummar S, Allen D, Monks A, et al. Cediranib for metastatic alveolar soft part sarcoma. J Clin Oncol 2013;31(18):2296-2302

60 Leu KM, Ostruszka LJ, Shewach D, et al. Laboratory and clinical evidence of synergistic cytotoxicity of sequential treatment with gemcitabine followed by docetaxel in the treatment of sarcoma. J Clin Oncol 2004;22(09):1706-1712

61 Grenader T, Goldberg A, Hadas-Halperin I, Gabizon A. Long-term response to pegylated liposomal doxorubicin in patients with 
metastatic soft tissue sarcomas. Anticancer Drugs 2009;20(01): $15-20$

62 Demetri GD, von Mehren M, Jones RL, et al. Efficacy and safety of trabectedin or dacarbazine for metastatic liposarcoma or leiomyosarcoma after failure of conventional chemotherapy: results of a phase III randomized multicenter clinical trial. J Clin Oncol 2016;34(08):786-793

63 Schöffski P, Chawla S, Maki RG, et al. Eribulin versus dacarbazine in previously treated patients with advanced liposarcoma or leiomyosarcoma: a randomised, open-label, multicentre, phase 3 trial. Lancet 2016;387(10028):1629-1637

64 van der Graaf WTA, Blay J-Y, Chawla SP, et al; EORTC Soft Tissue and Bone Sarcoma Group PALETTE study group. Pazopanib for metastatic soft-tissue sarcoma (PALETTE): a randomised, double-blind, placebocontrolled phase 3 trial. Lancet 2012;379(9829):1879-1886

65 Gounder MM, Mahoney MR, Van Tine BA, et al. Sorafenib for advanced and refractory desmoid tumors. N Engl J Med 2018;379 (25):2417-2428

66 Doebele RC, Drilon A, Paz-Ares L, et al; trial investigators. Entrectinib in patients with advanced or metastatic NTRK fusion-positive solid tumours: integrated analysis of three phase 1-2 trials. Lancet Oncol 2020;21(02):271-282

67 Drilon A, Laetsch TW, Kummar S, et al. Efficacy of larotrectinib in TRK fusion-positive cancers in adults and children. N Engl J Med 2018;378(08):731-739

68 Tawbi HA, Burgess M, Bolejack V, et al. Pembrolizumab in advanced soft-tissue sarcoma and bone sarcoma (SARC028): a multicentre, two-cohort, single-arm, open-label, phase 2 trial. Lancet Oncol 2017;18(11):1493-1501

69 Epizyme. Investors \& Media. Epizyme Announces U.S. FDA accelerated approval of TAZVERIKTM (tazemetostat) for the treatment of patients with epithelioid sarcoma. 2020. Accessed October 13, 2021 at: https://epizyme.gcs-web.com/news-releases/newsrelease-details/epizyme-announces-us-fda-accelerated-approval-tazveriktm-0

70 Blay J-Y, Chawla SP, Ray-Coquard I, et al. Phase III, placebocontrolled trial (SUCCEED) evaluating ridaforolimus as maintenance therapy in advanced sarcoma patients following clinical benefit from prior standard cytotoxic chemotherapy: long-term ( $\geq 24$ months) overall survival results. J Clin Oncol 2012;30(15, suppl):10010-10010

71 Mansfield AS, Murphy SJ, Harris FR, et al. Chromoplectic TPM3ALK rearrangement in a patient with inflammatory myofibroblastic tumor who responded to ceritinib after progression on crizotinib. Ann Oncol 2016;27(11):2111-2117

72 Chi Y, Yao Y, Wang S, et al. Anlotinib for metastasis soft tissue sarcoma: a randomized, double-blind, placebo-controlled and multi-centered clinical trial. J Clin Oncol 2018;36(15, suppl):11503-11503

73 Dickson MA, Schwartz GK, Keohan ML, et al. Progression-Free Survival Among Patients With Well-Differentiated or Dedifferentiated Liposarcoma Treated With CDK4 Inhibitor Palbociclib: A Phase 2 Clinical Trial. JAMA Oncol 2016 Jul 1;2(07): 937-940

74 Gounder MM, Somaiah N, Attia S, et al. Phase 2 results of selinexor in advanced de- differentiated (DDLS) liposarcoma (SEAL) study: a phase $2 / 3$, randomized, double blind, placebo controlled cross-over study. J Clin Oncol 2018;36(15, suppl):11512-11512

75 Patel SR, Zagars GK, Pisters PWT. The follow-up of adult soft-tissue sarcomas. Semin Oncol 2003;30(03):413-416 